\title{
A SLOWLY EXPANDING DISK AND FAST BIPOLAR OUTFLOW FROM THE S STAR $\pi^{1}$ GRUIS Po-Jian Chiu, ${ }^{1,2}$ Chi-Thiem Hoang, ${ }^{2}$ Dinh-V-Trung, ${ }^{2}$ Jeremy Lim, $^{2}$ Sun Kwok, ${ }^{2}$ Naomi Hirano, ${ }^{2}$ and C. Muthu ${ }^{2}$ Received 2005 October 18; accepted 2006 March 15
}

\begin{abstract}
We study the molecular outflow of the nearby evolved S star $\pi^{1}$ Gru. We imaged the outflow in CO $J=2-1$ and dust continuum with the Submillimeter Array. The CO emission was detected over a very broad velocity width of $\sim 90 \mathrm{~km} \mathrm{~s}^{-1}$. Our high-resolution images show that the outflow at low velocities $\left(\leq 15 \mathrm{~km} \mathrm{~s}^{-1}\right)$ is elongated eastwest and at high velocities $\left(\geq 25 \mathrm{~km} \mathrm{~s}^{-1}\right)$ is displaced north (at redshifted velocities) and south (blueshifted velocities) of center as defined by the dust continuum source. We model the spatial-kinematic structure of the lowvelocity outflow as a flared disk with a central cavity of radius $200 \mathrm{AU}$ and an expansion velocity of $11 \mathrm{~km} \mathrm{~s}^{-1}$, inclined by $55^{\circ}$ to our line of sight. We attribute the high-velocity component to a bipolar outflow that emerges perpendicular to this disk with a velocity of up to $\sim 45 \mathrm{~km} \mathrm{~s}^{-1}$. This high-velocity outflow may play an important role in shaping the gas envelope previously ejected by the AGB star and thus produce a bipolar morphology when the object evolves into a proto-planetary nebula.
\end{abstract}

Subject headings: circumstellar matter — stars: AGB and post-AGB — stars: individual ( $\pi$ Gruis)

\section{INTRODUCTION}

Near the end of their lives, intermediate-mass stars $\left(1 M_{\odot} \leq\right.$ $\left.M_{\text {star }} \leq 8 M_{\odot}\right)$ evolve through a relatively brief phase on the asymptotic giant branch (AGB) when they expel a significant fraction of their mass in the form of dusty molecular wind. This mass loss is driven by radiation pressure acting on dust grains formed above the stellar photosphere. The inferred mass-loss rates range from $10^{-7}$ to $10^{-5} M_{\odot} \mathrm{yr}^{-1}$ and may be even higher toward the terminal stages, leading to the removal of most of the hydrogen-rich envelope during the AGB phase. As a result, AGB stars are surrounded by a thick circumstellar envelope of gas and dust. This envelope is expected to be spherically symmetric, as the central stars are likely spherical and the radiation pressure, which is responsible for driving the mass loss, is isotropic. Highresolution imaging with the IRAM Plateau de Bure interferometer suggests that, indeed, most ( 70\%) AGB stars have approximately spherically symmetric envelopes (Neri et al. 1998). After the cessation of enhanced mass loss, AGB stars enter a shortlived transition phase called the proto-planetary nebula phase (PPN; see Kwok 1993). In this phase, the core of the AGB star contracts and evolves rapidly toward higher temperature with constant luminosity. Once the core becomes hot enough $\left(T_{\text {eff }}=\right.$ $30,000 \mathrm{~K})$ to produce significant amount of hard ultraviolet (UV) photons, it ionizes the surrounding envelope to produce a planetary nebula (PN). PNs are known exhibit a variety of morphologies ranging from circular to elliptical to bipolar (Balick \& Frank 2002). The origin of the morphological transformation from a near-spherical AGB envelope to, especially, bipolar PNs is unclear, although the discovery of bipolar PPNs suggests that this transition occurs early in the PPN phase or perhaps even earlier at the end of the AGB phase (Kwok et al. 1996, 1998). The detection of the earliest signatures of bipolar structures is therefore important in understanding the physical mechanisms responsible for shaping PNs.

\footnotetext{
1 Institute of Astronomy, National Central University, Number 300, Jhongda Road, Jhongli City, Taoyuan County 320, Taiwan

2 Institute of Astronomy and Astrophysics, Academia Sinica, P.O. Box 23141, Taipei 106, Taiwan.
}

The object $\pi^{1}$ Gru is a bright $\left(L_{\mathrm{bol}}=7450 L_{\odot}\right.$; Van Eck et al. 1998) AGB star with an S spectral type, i.e., C/O abundance ratio slightly below unity (Piccirillo 1980; Scalo \& Ross 1976), with a highly enhanced abundance of $s$-process elements, at a distance of 153 pc (Perryman et al. 1997). Feast (1953) discovered a faint main-sequence star next to $\pi^{1}$ Gru with the same proper motion. Later observations showed this companion to be a G0 main-sequence star (Ake \& Johnson 1992) located at a projected distance of 2".7 ( $400 \mathrm{AU})$ and a position angle (P. A.) of $200^{\circ} .4$ from $\pi^{1}$ Gru (Proust et al. 1981). The star $\pi^{1}$ Gru is a strong infrared source with an extended dust envelope. From Infrared Astronomical Satellite (IRAS) data, Young et al. (1993) infer a dust envelope of $0.28 \mathrm{pc}$ in size. Furthermore, with an expansion velocity of $15 \mathrm{~km} \mathrm{~s}^{-1}$ for its gas envelope as derived from $\mathrm{CO}$ observations, they inferred that the central star has been losing mass continuously for the last 21,000 yr. Sahai (1992, hereafter S92), observed $\pi^{1}$ Gru in CO $J=1-0$ and $J=2-1$ with the $15 \mathrm{~m}$ Swedish-ESO Submillimetre Telescope and found that the $\operatorname{CO} J=2-1$ line exhibits an unusual asymmetric doublehorned profile. The map of $\mathrm{CO} J=2-1$ emission with an angular resolution of $24^{\prime \prime}$ shows that the envelope is elongated in the east-west direction, has a size of at least $40^{\prime \prime}$, and exhibits a velocity gradient in the north-south direction. S92 suggested the presence of two kinematic components: a slow wind with a velocity of $\sim 15 \mathrm{~km} \mathrm{~s}^{-1}$, attributed to the double-horned feature in the $\mathrm{CO}$ line profiles, and a fast bipolar outflow with a velocity of $\geq 38 \mathrm{~km} \mathrm{~s}^{-1}$.

The outflow of $\pi^{1}$ Gru was subsequently studied at higher sensitivity by Knapp et al. (1999, hereafter K99), in CO $J=$ $2-1$ and $\mathrm{SiO} J=6-5$ using the Caltech Submillimeter Observatory (CSO) $10 \mathrm{~m}$ telescope. The CO $J=2-1$ line profile was found to be even broader than that measured by S92, indicating an outflow velocity of at least $70 \mathrm{~km} \mathrm{~s}^{-1}$. The angular resolution of the observation $\left(\sim 30^{\prime \prime}\right)$, however, is not adequate to resolve details in the envelope of $\pi^{1}$ Gru. K99 presented a model consisting of a tilted expanding disk to explain the doublehorned line profile and suggested that a faster wind is ejected along the poles of this disk. To better study the outflow structure of $\pi^{1}$, we imaged $\pi^{1}$ Gru in CO $J=2-1$ at high angular resolution with the Submillimeter Array (SMA). Because of its southern 
declination, $\pi^{1}$ Gru is not accessible to other northern hemisphere millimeter arrays.

\section{OBSERVATIONS AND DATA REDUCTION}

We observed $\pi^{1}$ Gru $\left[\alpha(\mathrm{J} 2000)=22^{\mathrm{h}} 22^{\mathrm{m}} 44^{\mathrm{s}} .2, \delta(\mathrm{J} 2000)=\right.$ $-45^{\circ} 56^{\prime} 52^{\prime \prime}$.6] on 2004 September 25 in CO $J=2-1$ and dust continuum in the $230 \mathrm{GHz}$ band with the SMA (Ho et al. 2004). The SMA consists of eight 6 m antennas located on Mauna Kea, Hawaii. The primary beam of the SMA antennas at $230 \mathrm{GHz}$ is $\sim 55^{\prime \prime}$, which is comparable with the total extent of the envelope of $\pi^{1}$ Gru mapped by S92 and K99. We configured the SMA correlator to cover a bandwidth of $2 \mathrm{GHz}$ with an effective frequency resolution of $0.8125 \mathrm{MHz}$, which corresponds to a velocity resolution of $1.0 \mathrm{~km} \mathrm{~s}^{-1}$. The wide frequency coverage of the SMA allows simultaneous observations of the CO $J=2-1$ line in the upper sideband and the $\mathrm{C}^{18} \mathrm{O}$ and ${ }^{13} \mathrm{CO}$ $J=2-1$ lines in the lower sideband. As we did not detect ${ }^{13} \mathrm{CO} J=$ $2-1$ and $\mathrm{C}^{18} \mathrm{O} J=2-1$, here we restrict our discussion to ${ }^{12} \mathrm{CO}$ $J=2-1$ emission.

The weather during the observation was excellent, with zenith opacity at $230 \mathrm{GHz}$ ranging from 0.059 to 0.075 . In its compact configuration, the SMA provides 28 independent baselines covering the $u-v$ plane from $\sim 6$ to $83 \mathrm{k} \lambda$, so that we are blind to structures larger than $\sim 40^{\prime \prime}$. Because $\pi^{1}$ Gru lies at a very low declination, the synthesized beam is elongated in the north-south direction. The nearby quasar 2258-279 was used for amplitude and phase calibration, and Uranus was used for bandpass and absolute flux calibration. The calibration was performed offline using the MIR package. We subsequently used the NRAO AIPS package for imaging and deconvolution. The images shown were made using robust weighting of the visibilities, with the robust parameter set to 0 as a compromise between minimizing the sidelobes and optimizing the sensitivity. The resulting synthesized beam has a size of $2^{\prime \prime} .1 \times 4^{\prime \prime} .2$ at a P.A. of $\sim 22^{\circ} .5$. We smoothed the $\mathrm{CO}$ data to a resolution of $1.625 \mathrm{MHz}$, corresponding to a velocity resolution of $\sim 2 \mathrm{~km} \mathrm{~s}^{-1}$. The resulting rms noise level in CO $J=$ $2-1$ is $0.08 \mathrm{Jy} \mathrm{beam}^{-1}$. The continuum image was obtained by averaging the data over all the line-free channels in the upper sideband and has a rms noise level of $3 \mathrm{mJy}^{\text {beam }^{-1}}$.

\section{RESULTS}

We detected an unresolved continuum source at $230 \mathrm{GHz}$ with a flux density of $22 \pm 3 \mathrm{mJy}$ at the stellar position determined by Hipparcos. The expected continuum flux from $\pi^{1} \mathrm{Gru}$, which is assumed to be a blackbody with effective temperature of $3000 \mathrm{~K}$ (Abia \& Wallerstein 1998) and a radius of $2.2 \times 10^{13} \mathrm{~cm}$ as inferred from the bolometric luminosity, is estimated to be $\sim 34 \mathrm{mJy}$. The difference $(\sim 35 \%)$ between observed and expected continuum flux at $230 \mathrm{GHz}$ may be due to uncertainties in the estimate of the stellar effective temperature, as a slightly higher temperature of $3300 \mathrm{~K}$ would make the expected continuum flux consistent with our result. In addition, the uncertainty in the absolute flux calibration, which might be significant especially for very low declination sources like $\pi^{1} \mathrm{Gru}$, may contribute to this difference between the observed and expected continuum flux.

We detected $\mathrm{CO} J=2-1$ emission over the velocity range from -60 to $35 \mathrm{~km} \mathrm{~s}^{-1}$, corresponding to $\pm 45 \mathrm{~km} \mathrm{~s}^{-1}$ from the systemic velocity of $V_{\mathrm{LSR}}=-11.8 \mathrm{~km} \mathrm{~s}^{-1}$. The spatially integrated line profile of the CO $J=2-1$ emission is shown in Figure 1 and can be seen to comprise a double-horned feature spanning the velocity range from -26 to $4 \mathrm{~km} \mathrm{~s}^{-1}$ with wings extending up to $-55 \mathrm{~km} \mathrm{~s}^{-1}$ in the blueshifted part and $35 \mathrm{~km} \mathrm{~s}^{-1}$ in the redshifted part (i.e., $\sim 30 \mathrm{~km} \mathrm{~s}^{-1}$ beyond the double-horned profile).

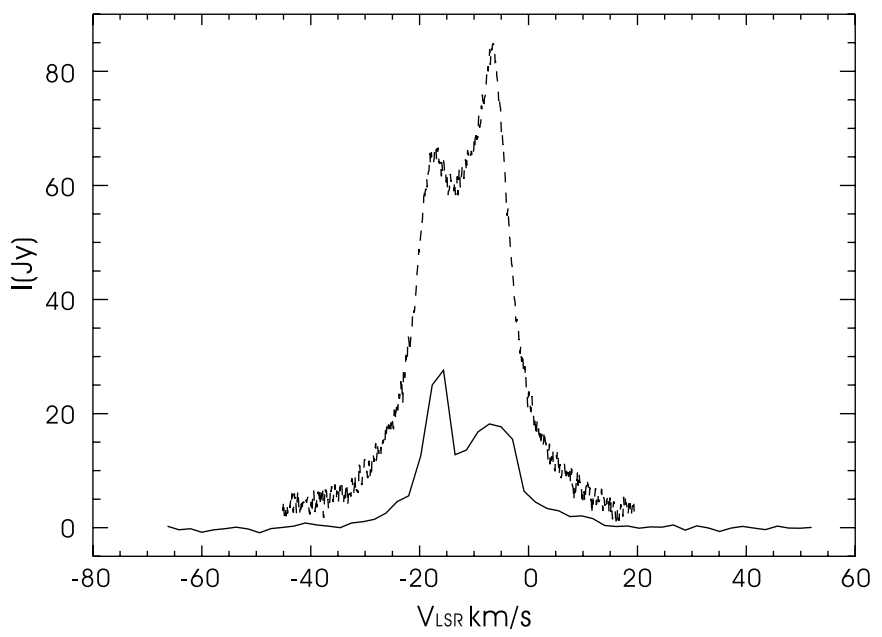

FIG. 1.-Solid line: $\mathrm{CO} J=2-1$ total flux line profile observed with the SMA at a resolution of $2 \mathrm{~km} \mathrm{~s}^{-1}$. The emission has an asymmetric doublehorned line profile. The dashed line shows the single-dish CO $J=2-1$ line profile from K99 for comparison.

The faint emission at very high velocities (up to blueshifted velocity of $-100 \mathrm{~km} \mathrm{~s}^{-1}$ and redshifted velocities of $80 \mathrm{~km} \mathrm{~s}^{-1}$ ) seen by K99 is not detected in our data. In addition the peak emission seen by SMA is shifted to the blue side of the profile in compared to single-dish data of K99. We also find that only $\sim 35 \%$ of the line intensity is detected within the double-horned profile and $\sim 20 \%$ in the high-velocity wings over the same velocity range. We attribute this large missing flux to the large size of the $\pi^{1}$ Gru envelope, which has been shown by the single-dish maps of S92 and K99 to be larger than 40". Such an extended envelope would be heavily overresolved by the SMA, which is more sensitive to structures on smaller scales. Indeed, our model for the envelope structure indicates that we would recover only $\sim 50 \%$ of the line intensity within the double-horned profile (see below), comparable with that detected in our observation. In the case of a homogeneous and smooth envelope, the missing flux due to incomplete $u-v$ coverage of the SMA data is expected to be similar for velocities around the line center, resulting in a similar total intensity line shape as observed with a single dish. Therefore, the shift in peak intensity of the integrated CO $J=2-1$ emission from red to blue velocity in comparison to that observed by K99 is most likely the result of overresolving the extended and inhomogeneous envelope of $\pi^{1} \mathrm{Gru}$.

The channel maps of the CO $J=2-1$ emission are shown in Figure 2. The morphology is clearly inconsistent with a spherically expanding envelope, which would exhibit a circular or ring morphology with decreasing radius with increasing velocity away from the systematic velocity. Instead, we find that emission at and near the systemic velocity $\left(-11 \mathrm{~km} \mathrm{~s}^{-1}\right)$ has a flattened morphology that flares out in the east-west direction. At higher velocities of $\sim 10-15 \mathrm{~km} \mathrm{~s}^{-1}$ from the systemic velocity, the emission becomes more compact and shows a clear spatial shift between the redshifted (receding) and blueshifted (approaching) parts. The redshifted part of the envelope is located to the north and the blueshifted part to the south of the stellar position as defined by the continuum source. As described in $\S 4$, we model these low-velocity parts of the channel maps as an inclined equatorially enhanced outflow (hereafter "flared disk"). In the central three channels (at velocities $-9.3,-11.4$, and $-13.5 \mathrm{~km} \mathrm{~s}^{-1}$ ), the $\mathrm{CO}$ emission does not peak at the center but instead on either side to the east and west of the central star. As shown also in $\S 4$, this can be explained by the presence of a 


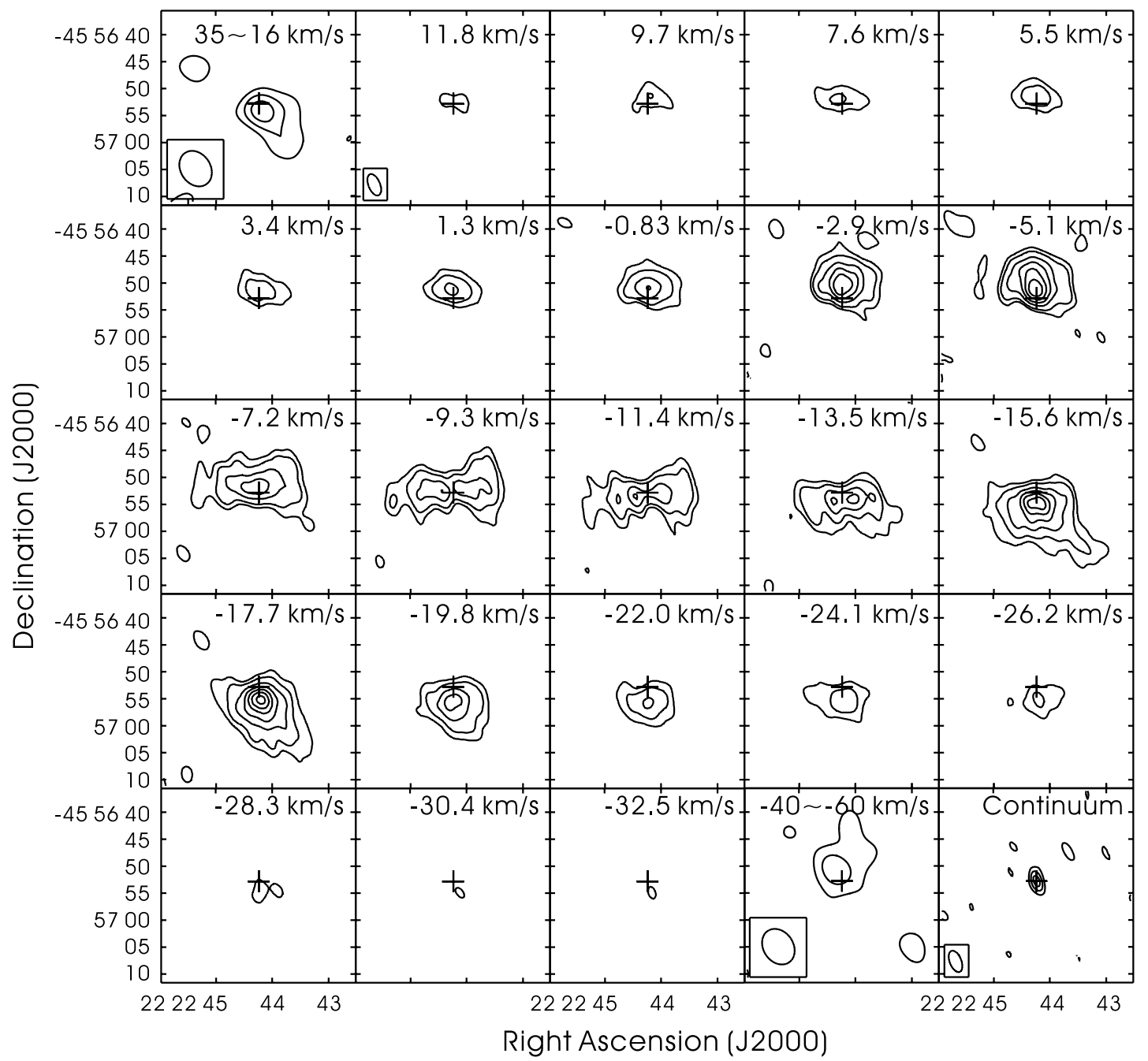

FIG. 2-Channel maps of the $\mathrm{CO} J=2-1$ emission from $\pi^{1}$ Gru. The synthesized beam is shown in the lower left corner and has a size of 2 "' $2 \times 44^{\prime \prime} 2$ for the lower velocity range -32.5 to $11.8 \mathrm{~km} \mathrm{~s}^{-1}$ and $5^{\prime \prime} \times 7^{\prime \prime}$ for the higher velocity ranges -60 to -40 and $16-35 \mathrm{~km} \mathrm{~s}^{-1}$ (see text). The contours are plotted at $2,4,8,12,16$, $20 \times 0.2 \mathrm{Jy} \mathrm{beam}^{-1}$ for the lower velocity range, and 2, 4, 6, $8 \times 0.04 \mathrm{Jy}_{\text {beam }}{ }^{-1}$ for the high-velocity ranges, where the lowest contour is at twice the rms noise level. The bottom right frame shows the continuum emission at $230 \mathrm{GHz}$ with contour levels at $2,4,6,8 \times 0.003 \mathrm{Jy} \mathrm{beam}^{-1}$.

cavity or depression at the center of the expanding disk. Note the weak extensions to the southwest of the disk at velocities -15.6 and $-17.7 \mathrm{~km} \mathrm{~s}^{-1}$ and the east side of the disk at velocities -7.2 and $-9.3 \mathrm{~km} \mathrm{~s}^{-1}$. These features may trace the outer regions of a largely overresolved envelope or may be artifacts produced by the sidelobes of the telescope.

To improve our sensitivity to the weak high-velocity components in the velocity range $25-45 \mathrm{~km} \mathrm{~s}^{-1}$ from the systemic velocity, we average the data over a larger velocity range of $\sim 6 \mathrm{~km} \mathrm{~s}^{-1}$ per channel and downweighted the long baselines to produce a larger synthesized beam of $5^{\prime \prime} \times 7^{\prime \prime}$. This high-velocity component exhibits a clear bipolarity with the blueshifted emission to the north and redshifted emission to the south of the star, opposite to that of the low-velocity component. Figure 2 shows the high-velocity component integrated over the velocity range $16-35 \mathrm{~km} \mathrm{~s}^{-1}$ and -40 to $-60 \mathrm{~km} \mathrm{~s}^{-1}$. We interpret this highvelocity emission as a bipolar outflow perpendicular to the disk plane.

In Figure 3, we plot the velocity of the emission as a function of position along the north-south direction. The emission at velocity $\left|V-V_{\text {sys }}\right| \leq 15 \mathrm{~km} \mathrm{~s}^{-1}$ corresponds to the disk, and $\Delta V \geq 25 \mathrm{~km} \mathrm{~s}^{-1}$ corresponds to the bipolar outflow as described above. Emission with $\Delta V \leq 5 \mathrm{~km} \mathrm{~s}^{-1}$ is located close to the cen- ter, whereas emission with $\Delta V=5-15 \mathrm{~km} \mathrm{~s}^{-1}$ are spatially extended to the north and south, respectively, at redshifted and blueshifted velocities. On the other hand, emission at $\Delta V \geq 25 \mathrm{~km} \mathrm{~s}^{-1}$ has the opposite pattern with blueshifted emission to the north and redshifted to the south, and seems to exhibit a trend of increasing velocity with increasing radial distance. Figure 4 shows the integrated intensity map of the redshifted and blueshifted components of the high-velocity outflow (contour) superposed on the intensity-weighted mean-velocity map of the disk (color). The disk exhibits a velocity gradient in the north-south direction, whereas the high-velocity outflow shows the opposite pattern. A schematic diagram of these two components that we propose can explain our results is shown in Figure 5.

\section{A MODEL FOR THE $\pi^{1}$ GRU ENVELOPE}

We can separate the envelope of $\pi^{1}$ Gru into two components based on its spatial-kinematic structure: a slow equatorially enhanced wind in the form of a flared disk and a high-velocity outflow orthogonal to this disk. To gain a more detailed understanding of the expanding disk, we construct a simple model similar to that used by Bujarrabal et al. (1997). At each point inside the disk the excitation of $\mathrm{CO}$ molecules is calculated using the large velocity gradient (LVG) approximation. Because the 


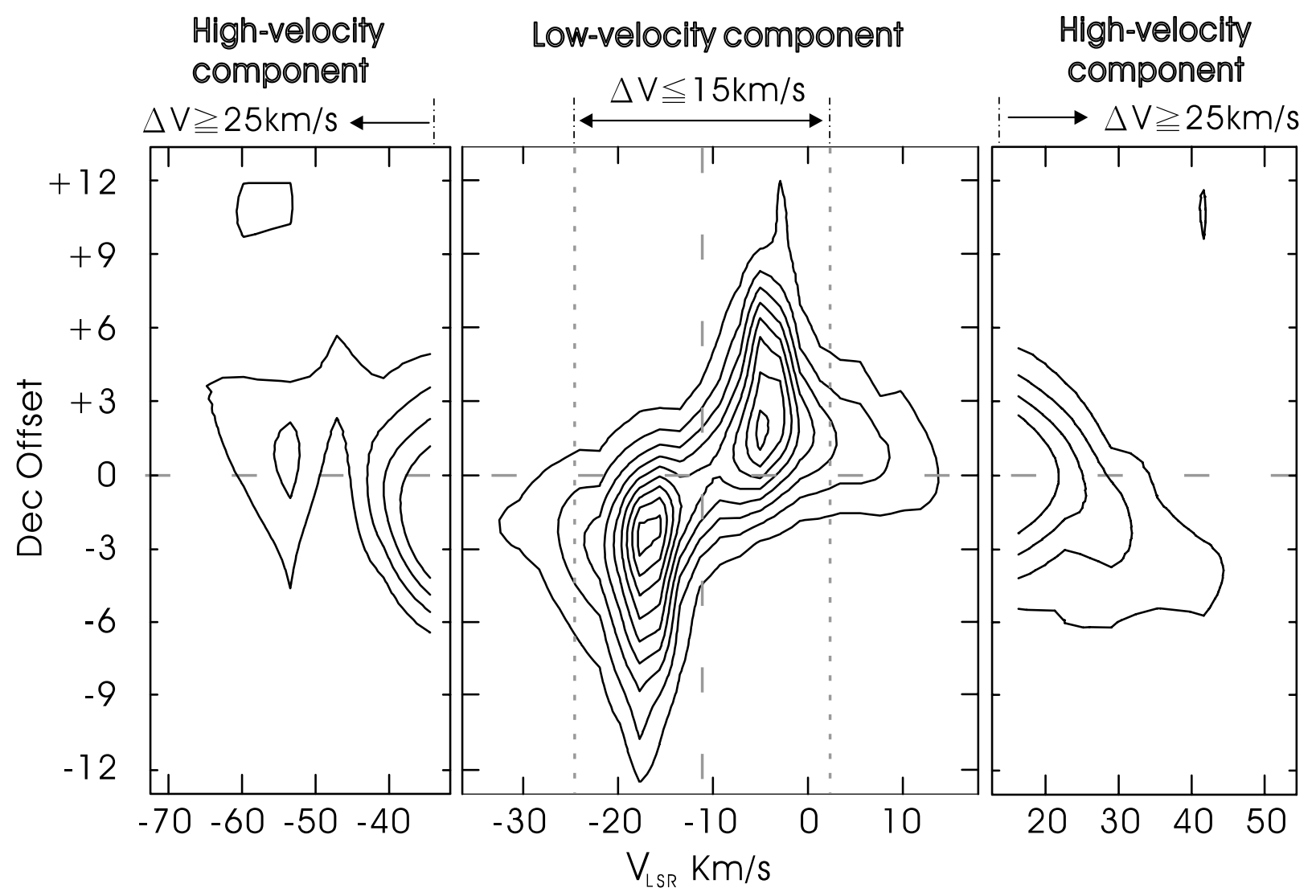

Fig. 3.-Position-velocity diagram along a north-south cut through the stellar position. Middle: Emission at velocities of -35 to $15 \mathrm{~km} \mathrm{~s}{ }^{-1}$. The velocity resolution is $2 \mathrm{~km} \mathrm{~s}^{-1}$, and the contour levels are plotted at $0.2,0.5,0.8,1.1,1.4,1.7,2.0$, and $2.3 \mathrm{Jy} \mathrm{beam}^{-1}$. Left and right: Emission at higher velocities of -72 to $-35 \mathrm{~km} \mathrm{~s}^{-1}$ (left) and $15-53 \mathrm{~km} \mathrm{~s}^{-1}$ (right). The velocity resolution is $6 \mathrm{~km} \mathrm{~s}^{-1}$, and the contour levels are plotted at $1,4,7,10,13 \times 0.04 \mathrm{Jy} \mathrm{beam}{ }^{-1}$.

expansion velocity of the disk is much larger than the local linewidth, the use of LVG is justified. In the excitation calculations we take into account 11 rotational levels of the $\mathrm{CO}$ molecule. The local absorption and emissivity of rotational lines are then derived from the level populations and the local linewidth, which is determined by both turbulence and thermal motion. The $\mathrm{CO}$ $J=2-1$ intensity at different lines of sight is then calculated by integrating directly the standard radiative transfer equation. The channel maps produced by our model differ from those produced by our observations that largely resolved out the outer regions of the extended envelope. To account for this, we use the maps of the predicted CO $J=2-1$ intensity at each velocity and corrected for the primary beam response of the SMA antennas as an input to the program uvmodel in the MIRIAD package to generate visibilities that would be measured by the SMA for the same $u-v$ coverage. The model visibilities are then processed in the same fashion as the real SMA data to produce the final model channel maps.

At and near the systemic velocity in the ${ }^{12} \mathrm{CO} J=2-1$ channel maps (Fig. 2), the presence of emission peaks not at the stellar position but on either side of the star requires a central depression or cavity in the disk. The geometry of the disk is then determined by the inner $\left(R_{\min }\right)$ and outer $\left(R_{\max }\right)$ radius, the thickness $Z$ at the inner radius, and an opening angle of $50^{\circ}$. We adopt the same outer radius of $5 \times 10^{16} \mathrm{~cm}$ as the disk inferred by K99. The kinetic temperature of the gas is assumed to follow a power law $T(r)=300 \mathrm{~K}\left(r / 10^{15} \mathrm{~cm}\right)^{-0.7}$, which is also used by $\mathrm{K} 99$ and is similar to that predicted in the AGB wind models of Goldreich \& Scoville (1976) and Kwan \& Linke (1982). We also adopt the systemic velocity $V_{\mathrm{LSR}}=-11.8 \mathrm{~km} \mathrm{~s}^{-1}$ and the same $\mathrm{CO}$ abundance respect to $\mathrm{H}_{2}\left(f=[\mathrm{CO}] /\left[\mathrm{H}_{2}\right]\right)$ of $6.5 \times 10^{-4}$, similar to that used by K99. The envelope is assumed to expand at a constant velocity of $11 \mathrm{~km} \mathrm{~s}^{-1}$. A local turbulent velocity of $2 \mathrm{~km} \mathrm{~s}^{-1}$ is also adopted in our model. The density of the gas at each grid point is calculated according to the prescribed massloss rate at the corresponding radius.

The best-fit model to both the CSO observation of K99 and the morphology of CO $J=2-1$ emission in our channel maps (at $\Delta V \leq 15 \mathrm{~km} \mathrm{~s}^{-1}$ ) is a flared disk with an opening angle of $50^{\circ}$. The inner radius of the disk $R_{\min }$ is determined to be $3 \times$ $10^{15} \mathrm{~cm}$ by matching the separation of the two emission peaks in the central velocity channels. The disk thickness $Z$ at the inner radius is $2 \times 10^{15} \mathrm{~cm}$. The inclination angle of the flared disk to the line of sight is $55^{\circ}$. We find that a constant mass-loss rate produces more compact $\mathrm{CO} J=2-1$ emission than is observed. A better agreement can be achieved if the mass-loss rate increases with radius according to the power law $\left(r / R_{\min }\right)^{0.25}$. We find that the mass-loss rate at the inner radius is $5 \times 10^{-7} M_{\odot} \mathrm{yr}^{-1}$. The parameters of our model for the expanding disk around $\pi^{1}$ Gru are summarized in Table 1. The decrease in mass-loss rate with time is not unexpected, as the presence of an inner depression or cavity already suggests that the mass-loss rate of $\pi^{1} \mathrm{Gru}$ has decreased dramatically over the last $90 \mathrm{yr}$. A comparison between the predicted $\mathrm{CO} J=2-1$ profiles at various positions within the envelope for a telescope beam of $30^{\prime \prime}$ and the CSO observations of K99 is shown in Figure 6.

As expected for an extended envelope, we predict that the SMA would recover less than $50 \%$ of the total CO $J=2-1$ flux of our model. The actual amount of missing flux in our map is slightly larger, $\sim 65 \%$, suggesting that the $\mathrm{CO} J=2-1$ emission 


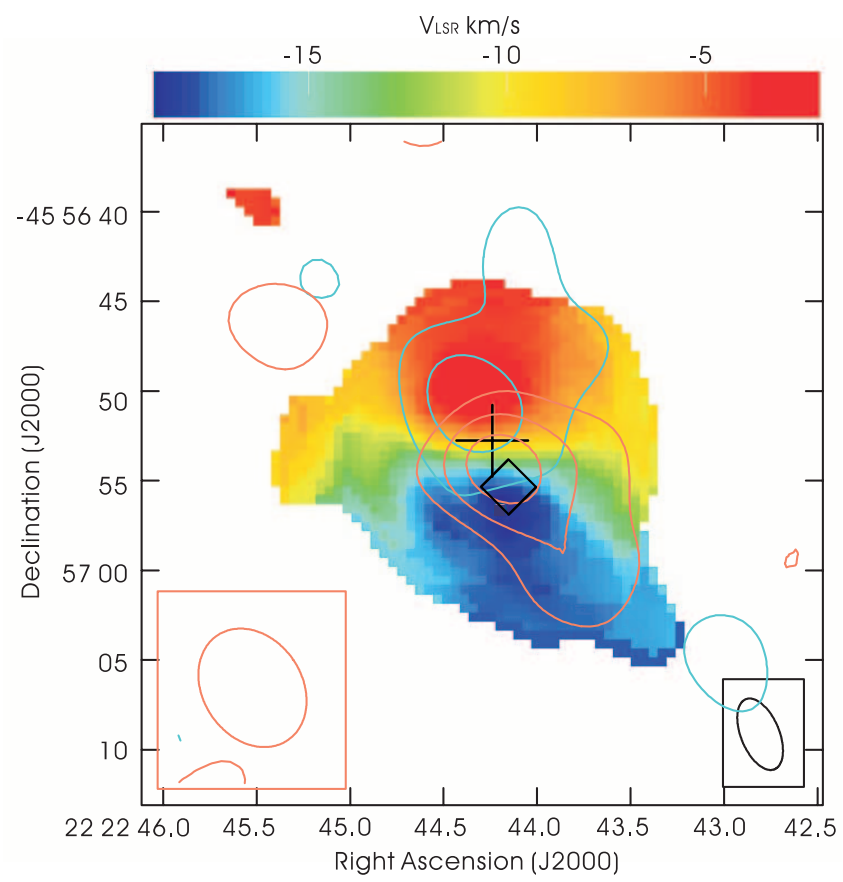

FIG. 4.-Intensity-weighted mean-velocity color map of the CO $J=2-1$ emission in the velocity range -20 to $0 \mathrm{~km} \mathrm{~s}^{-1}$. We propose that the component corresponds to an inclined disk. The integrated intensity contour map of the emission at higher velocities of 35-16 $\mathrm{km} \mathrm{s}^{-1}$ (redshifted component in red) and -60 to $-40 \mathrm{~km} \mathrm{~s}^{-1}$ (blueshifted component in blue). We propose this component corresponds to a bipolar outflow. The synthesized beams of the high- and low-velocity components are shown in the lower left and right corners, respectively. The cross and diamond indicate the locations of $\pi^{1}$ Gru and the companion, respectively.

is somewhat more extended than that predicted by our simple model. In Figure 7 we show the channel maps of the $\operatorname{CO} J=$ $2-1$ emission produced by our model. Both the shape of the envelope near the systemic velocity and the morphological change of the emission at higher velocity seen in our SMA maps of Figure 2 are reproduced by our model. The predicted intensityweighted mean-velocity map as shown in Figure 8 indicates a clear velocity gradient in the north-south direction as observed with the SMA (Fig. 4). The position-velocity diagram produced

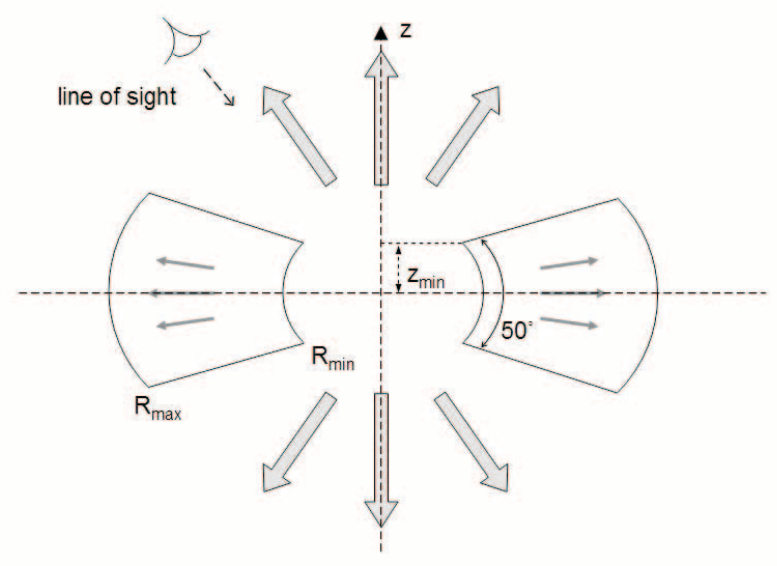

FIG. 5.-Schematic diagram of our model for the $\pi^{1}$ Gru envelope. A slowly expanding flared disk is inclined by $55^{\circ}$ to our line of sight. A high-velocity bipolar outflow is oriented perpendicular to this disk.
TABLE 1

Model Parameters of $\pi^{1}$ Gru Envelope

\begin{tabular}{|c|c|}
\hline Parameter & Value \\
\hline . & $11 \mathrm{~km} \mathrm{~s}^{-1}$ \\
\hline$V_{\mathrm{LSR}}$ & $-11.8 \mathrm{~km} \mathrm{~s}^{-1}$ \\
\hline 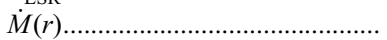 & $5 \times 10^{-7}\left(r / 3 \times 10^{15} \mathrm{~cm}\right)^{0.25} M_{\odot} \mathrm{yr}^{-1}$ \\
\hline$T_{k}(r) \ldots \ldots \ldots \ldots \ldots$ & $300 \mathrm{~K}\left(r / 10^{15} \mathrm{~cm}\right)^{-0.7}$ \\
\hline$f=[\mathrm{CO}] /\left[\mathrm{H}_{2}\right] \ldots \ldots \ldots \ldots \ldots \ldots \ldots \ldots$ & $6.5 \times 10^{-4}$ \\
\hline 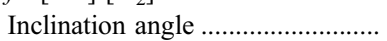 & $55^{\circ}$ \\
\hline 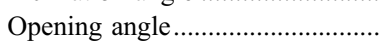 & $50^{\circ}$ \\
\hline 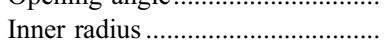 & $3 \times 10^{15} \mathrm{~cm}$ \\
\hline Inner thickness $Z \ldots \ldots \ldots \ldots \ldots \ldots \ldots \ldots$ & $2 \times 10^{15} \mathrm{~cm}$ \\
\hline
\end{tabular}

by our model also shown in Figure 8 is in good agreement with that observed (Fig. 3).

Although our simple flared-disk model can explain the main features (morphology and kinematics) of the $\pi^{1}$ Gru envelope at low velocities, the emission at higher velocity of $15 \mathrm{~km} \mathrm{~s}^{-1} \leq$ $\Delta V \leq 25 \mathrm{~km} \mathrm{~s}^{-1}$ cannot be accounted for by our simple model. Given that the emission at velocities $\geq 25 \mathrm{~km} \mathrm{~s}^{-1}$ is produced by a high-velocity bipolar outflow in the north-south direction, the emission at intermediate velocities $\left(15 \mathrm{~km} \mathrm{~s}^{-1} \leq \Delta V \leq\right.$ $25 \mathrm{~km} \mathrm{~s}^{-1}$ ) may originate from gas disturbed or entrained by this high-velocity outflow; note that the emission at intermediate velocities has the same sense in velocity gradient as the highvelocity bipolar outflow. In addition, our disk model does not predict the existence of the weak extended feature at velocities $-7.2,-9.3,-15.6$, and $-17.7 \mathrm{~km} \mathrm{~s}^{-1}$ in the channel maps. If these features are real, they may be part of the outer envelope (i.e., the envelope is not perfectly smooth in structure) or possibly parts of a different collimated outflow.

\section{DISCUSSION}

\subsection{Envelope and the Bipolar Outflow}

The low- and high-velocity components of the $\mathrm{CO} J=2-1$ emission show different distributions and kinematics. Based on our model, the spatial-kinematic structure of the low-velocity component $\left( \pm 15 \mathrm{~km} \mathrm{~s}^{-1}\right)$ can be explained by an equatorially enhanced wind in the form of a tilted (by $55^{\circ}$ ) and expanding $\left(11 \mathrm{~km} \mathrm{~s}^{-1}\right)$ flared disk with a central depression or cavity. The latter may be caused by a sudden decrease in the mass loss or carved out by the high-velocity bipolar outflow. Although the north-south displacement of the low-velocity emission in the channel maps resembles a bipolar outflow, it is in fact better explained as the approaching (front) and receding (back) parts of a tilted disk. The opposite kinematic orientation of the highvelocity component $\left(\Delta V \geq 25 \mathrm{~km} \mathrm{~s}^{-1}\right)$ compared with the lowvelocity component can be explained if this component is a bipolar outflow perpendicular to the disk plane with a deprojected velocity of at least $60 \mathrm{~km} \mathrm{~s}^{-1}$. As for the emission at intermediate velocities $\left(15 \mathrm{~km} \mathrm{~s}^{-1} \leq \Delta V \leq 25 \mathrm{~km} \mathrm{~s}^{-1}\right)$, which has the same sense in velocity gradient as the high-velocity bipolar outflow, this may be material punched out of the disk by the bipolar outflow. Alternatively, or in addition, the bipolar outflow may simply have a very large opening angle.

For an expansion velocity of $11 \mathrm{~km} \mathrm{~s}^{-1}$, we estimate a kinematic timescale for the flared disk of only $\sim 1450 \mathrm{yr}$. The mass and momentum of the disk according to our model are $5.7 \times$ $10^{-4} M_{\odot}$ and $\sim 1.3 \times 10^{36} \mathrm{~g} \mathrm{~cm} \mathrm{~s}^{-1}$, respectively. For the highvelocity bipolar outflow, we can estimate a lower limit for its mass from the $\mathrm{CO}$ observation with the $\mathrm{CSO}$. Assuming local 


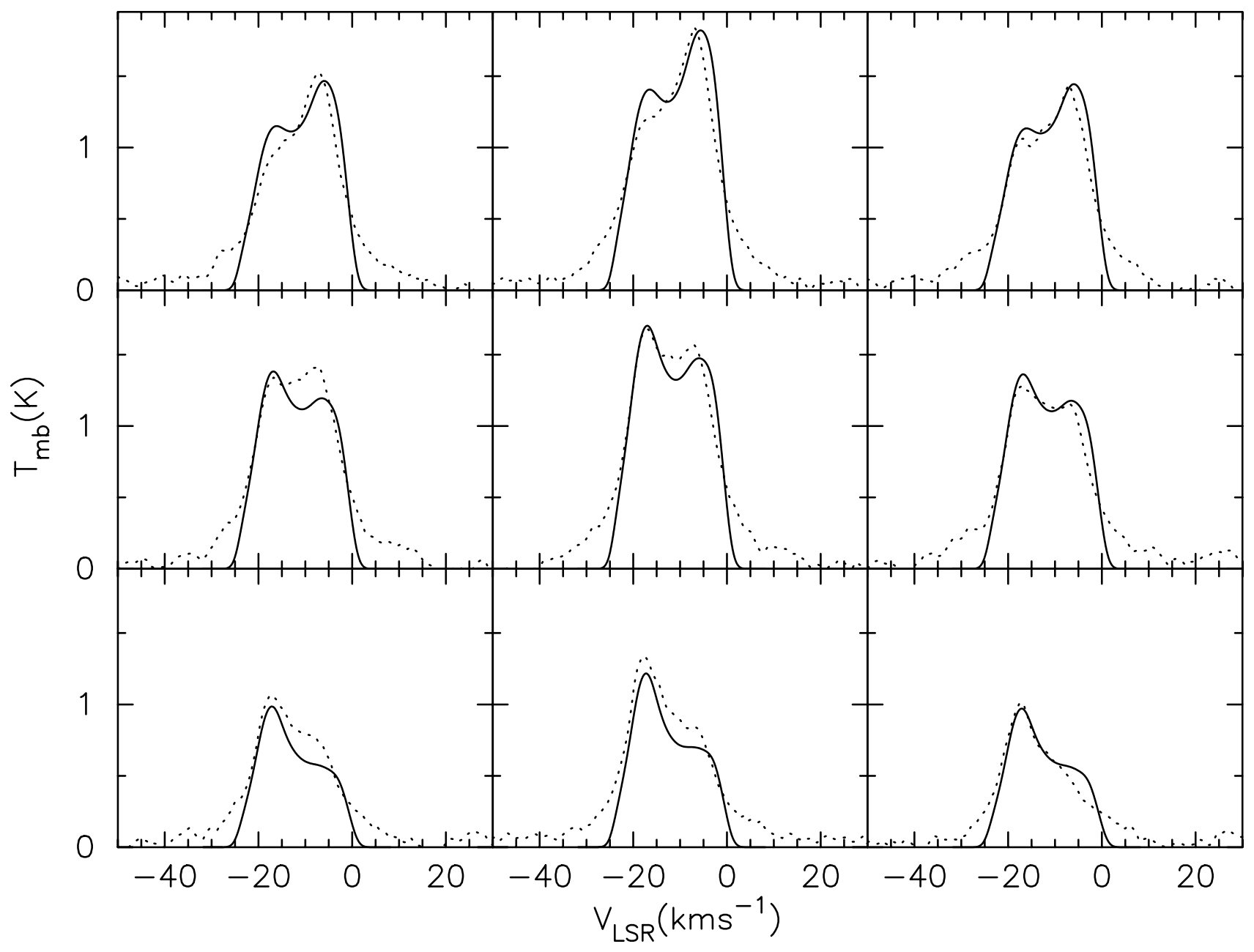

FIG. 6.-Comparison between our model predictions (solid lines) and the CSO observation of $\pi^{1}$ Gru by K99 (dotted lines) for the CO $J=2-1$ line. The maps produced by our model have been convolved with the CSO beam size of $30^{\prime \prime}$. The central panel corresponds to the stellar position, and the grid spacing between panels is $10^{\prime \prime}$. Our model line profiles take into account a pointing offset of $+5^{\prime \prime}$, as suggested by K99.

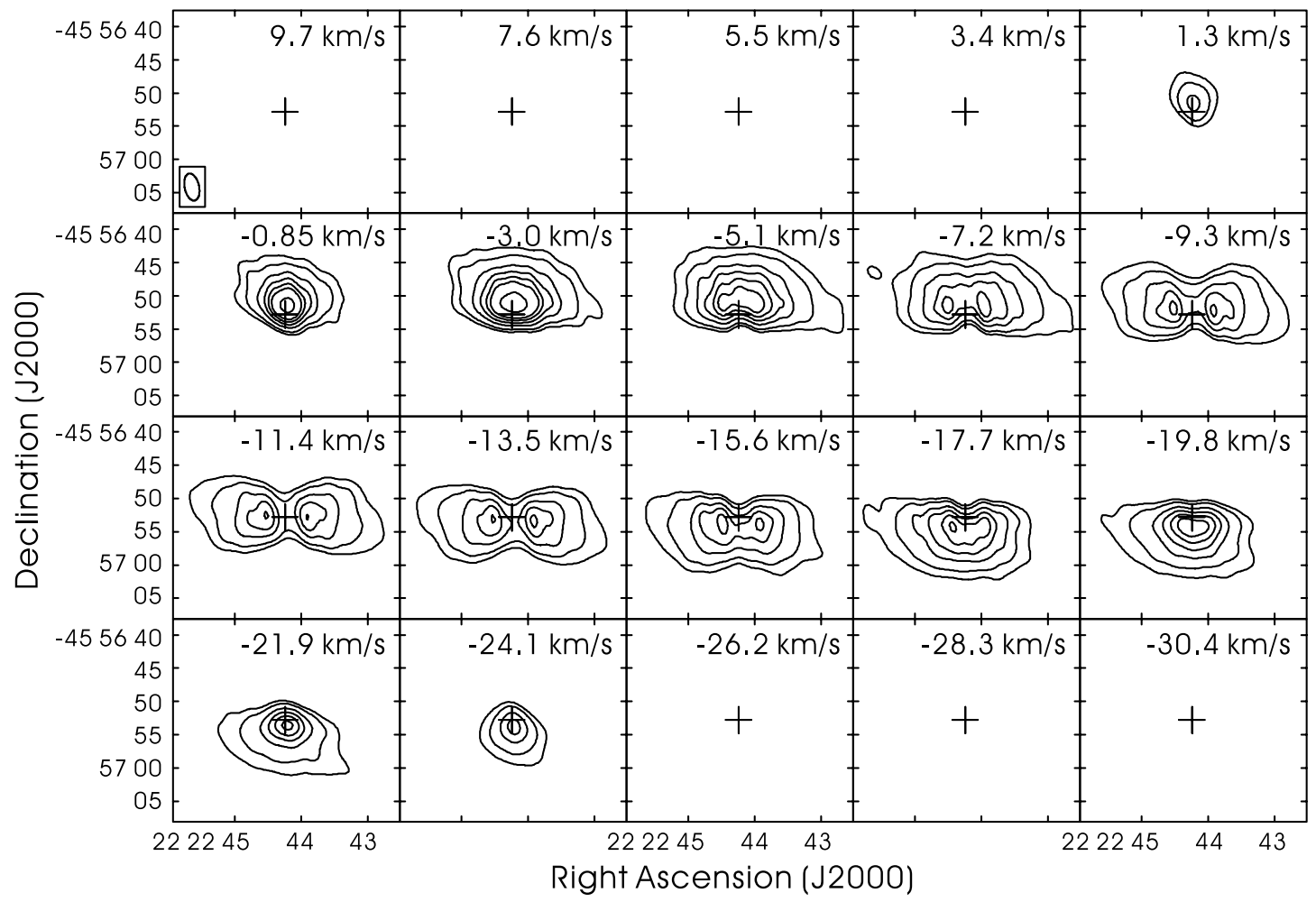

FIG. 7.- Channel maps of $\mathrm{CO} J=2-1$ emission from our model of an inclined expanding disk as observed with the same $u$ - $v$ coverage as our observation of $\pi^{1}$ Gru with the SMA. The synthesized beam is $2^{\prime \prime} \times 4^{\prime \prime}$. Contour levels are plotted at 2, 4, 8, 12, 16, 20, $30 \times 0.2 \mathrm{Jy} \mathrm{beam}^{-1}$. 

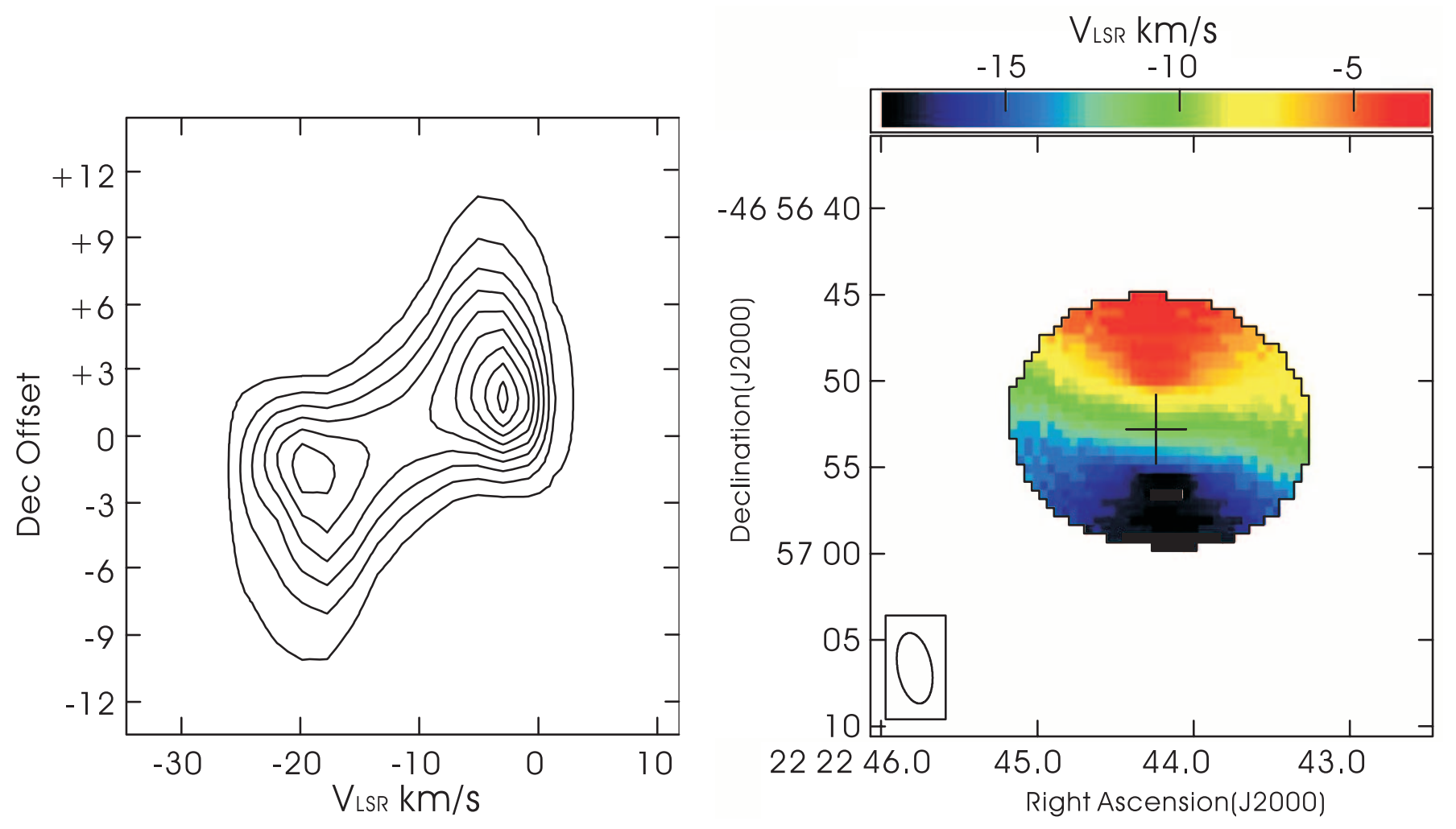

FIG. 8. - Position-velocity diagram along a north-south cut through the stellar position (left) and intensity-weighted mean-velocity map (right) from our model. The synthesized beam is $2^{\prime \prime} \times 4^{\prime \prime}$. Contour levels are plotted at $1,4,10,13,16,19,22,25 \times 0.2 \mathrm{Jy}_{\text {beam }}^{-1}$.

thermodynamic equilibrium and optically thin emission, we use the formula (Olofsson \& Nyman 1999)

$$
M=\frac{16 \pi k m_{\mathrm{H}}}{h c g_{u} A_{u l} f} \frac{D^{2}}{A_{e}} I Q\left(T_{\mathrm{ex}}\right) e^{E_{u} / k T_{\mathrm{ex}}},
$$

where $D$ is the distance, $f=[\mathrm{CO}] /[\mathrm{H}]$ as defined in $\S 4, A_{e}$ is the effective area of the telescope, $I=\int T_{\mathrm{mb}} d v$ is the integrated intensity over the velocity range from \pm 20 to $\pm 80 \mathrm{~km} \mathrm{~s}^{-1}$ from the systemic velocity, $Q$ is the partition function of CO molecules, $E_{u}$ is the energy of the upper level, and $T_{\mathrm{ex}}$ is the excitation temperature. Using $f=6.5 \times 10^{-4}$, as in our model, and $T_{\mathrm{ex}}=15 \mathrm{~K}$, similar to that used by Olofsson \& Nyman (1999), we obtain a mass for the high-velocity bipolar outflow of $M_{\text {outflow }} \sim 5.7 \times 10^{-5} M_{\odot}$ and a momentum of $P_{\text {outflow }}=$ $\int m(v) v / \sin (55) d v \sim 4.7 \times 10^{35} \mathrm{~g} \mathrm{~cm} \mathrm{~s}^{-1}$. Furthermore, assuming that the high-velocity outflow is perpendicular to the disk, with a projected angular extent from the star of $\sim 2^{\prime \prime}$ we estimate a kinematic timescale for the bipolar outflow of only $\sim 60 \mathrm{yr}$. Comparing the momentum with per unit time $P / \Delta t$ (where $\Delta t$ is the kinematic timescale) of the disk and bipolar outflow with the momentum carried by the stellar radiation pressure per unit time $L / c$, we find a ratio $(P / \Delta t) /(L / c) \sim 0.02$ for the disk and $\sim 0.26$ for the outflow. This indicates, but does not imply, that both the disk and outflow could be driven by the radiation pressure from the central star.

Another carbon star, V Hya, also possesses an asymmetric double-horned line profile and very broad line wings similar to $\pi^{1}$ Gru (Knapp et al. 1997). Single-dish molecular line observations have shown that the envelope of $\mathrm{V}$ Hya is elongated along the north-south direction with a velocity gradient along its minor axis (Kahane et al. 1996). Hirano et al. (2004) imaged the envelope of V Hya at high angular resolution using the SMA. They interpreted their results in terms of three separate components: a slowly expanding disk $\left(\Delta V \leq 8 \mathrm{~km} \mathrm{~s}^{-1}\right)$, a medium-velocity outflow $\left(8 \mathrm{~km} \mathrm{~s}^{-1} \leq \Delta V \leq 60 \mathrm{~km} \mathrm{~s}^{-1}\right)$, and a high-velocity bipolar outflow $\left(\Delta V \sim 60-160 \mathrm{~km} \mathrm{~s}^{-1}\right)$. The morphology and kinematics of its molecular envelope are remarkably similar to those of $\pi^{1} \mathrm{Gru}$. Around the systemic velocity, the emission shows an elongated structure with a velocity gradient along the minor axis. The high-velocity component is oriented perpendicular to this elongated structure with an opposite sense in velocity gradient. We believe that the model developed for $\pi^{1} \mathrm{Gru}$ also can be applied to V Hya. That is, the molecular envelope of $\mathrm{V}$ Hya comprises a slowly expanding disk with a velocity $\Delta V \approx \pm 20 \mathrm{~km} \mathrm{~s}^{-1}$, a high-velocity bipolar outflow with a velocity $\pm 60-160 \mathrm{~km} \mathrm{~s}^{-1}$, and gas at intermediate velocities comprising material punched out by the high-velocity bipolar outflow. The intermediate-velocity emission in V Hya, however, covers a velocity range of $40 \mathrm{~km} \mathrm{~s}^{-1}$ that is much wider than in $\pi^{1} \mathrm{Gru}$.

\subsection{Origin of the Disk-Outflow Structure in $\pi^{1}$ Gru}

The disk-outflow envelope structure of $\pi^{1}$ Gru and V Hya is not typical of AGB envelopes but is often seen in PPNs and PNs. For example, Kwok et al. (2000) detected a disk in scattered light in the PPN IRAS 17106-3046 that is attributed to collimating a bipolar outflow. In the case of $\pi^{1} \mathrm{Gru}$, however, the inner radius of the disk is so large that it cannot be the collimating agent.

The origin of a strong equatorial enhancement in mass loss in AGB envelope is not well understood. At least two mechanisms have been suggested for the formation of such disklike structures. Magnetohydrodynamic simulations suggest a moderate stellar dipole magnetic field can compress the molecular gas in the 
equatorial region and also produce a polar wind at higher velocity (Matt et al. 2000). Alternatively, an equatorially enhanced wind can be produced in a binary system. According to Morris (1987), Iben \& Livio (1993), and Mastrodemos \& Morris (1998, 1999), the gravitational perturbation of a companion star can concentrate the AGB wind in the orbital plane, as well as attract material to form an accretion disk around the companion. A fast polar wind powered and collimated by this accretion disk then clears out envelope material along the polar direction, thus producing a disk-outflow envelope structure.

Proust et al. (1981) showed that separation of $\pi^{1}$ Gru and its companion is 2 ".7 at a P. A. of $200^{\circ} .4$ (see Fig. 4). The projected distance of $400 \mathrm{AU}$ from $\pi^{1}$ Gru puts the secondary well outside the central cavity and possibly inside the expanding disk if its orbit and the disk are coplanar. If we assume that the secondary is located on the disk plane, with the latter inclined by $55^{\circ}$ to our line of sight, the deprojected separation of the binary system is $500 \mathrm{AU}$. Although the separation seems to be relatively large, the effect of the companion might still be important in focusing the wind from $\pi^{1}$ Gru toward the orbital plane and create significant density enhancement as predicted by Mastrodemos \& Morris (1999) for wide binary systems. On the other hand, the estimated separation is much larger than that required in the model of Morris (1987), and the density of the gas around the secondary is too low ( $\sim 10^{4} \mathrm{~cm}^{-3}$ in comparison to much higher gas density in simulations of Mastrodemos \& Morris [1998]) for accretion to operate efficiently. However, it is conceivable that the companion is in a highly elliptical orbit and that the system is currently close to its largest separation. At the closest approach, the secondary might capture enough gas from the denser surroundings to form an accretion disk and blow the high-velocity outflow. Alternatively, there might be a much closer companion that has so far escaped detection and currently is the source of the high-velocity outflow. In addition, the presence of the secondary within the expanding disk may trigger some kinds of inhomogeneity such as the spiral shock predicted by the smoothed particle hydrodynamics simulations of Theuns et al. (1996) or Mastrodemos \& Morris (1998,
1999). Unfortunately, our SMA data do not provide any clear evidence for these inhomogeneities. Future observations with higher sensitivity and better spatial coverage will be able to reveal the large-scale structure in the envelope of $\pi^{1} \mathrm{Gru}$ and thus probe the effect of the companion on the circumstellar envelope.

\section{CONCLUSION}

We have imaged at high angular resolution the ${ }^{12} \mathrm{CO} J=2-1$ emission of S star $\pi^{1}$ Gru. We interpret our results in terms of two distinct components: a slowly expanding equatorial wind with velocity $\leq 15 \mathrm{~km} \mathrm{~s}^{-1}$ and a high-velocity bipolar outflow with velocity up to $45 \mathrm{~km} \mathrm{~s}^{-1}$. We modeled the equatorial wind as a flared disk tilted by $55^{\circ}$ to our line of sight. The disk has a central cavity with a inner radius of $3 \times 10^{15} \mathrm{~cm}$ and an outer radius of $5 \times 10^{16} \mathrm{~cm}$. The opening angle of the disk is $50^{\circ}$, and its expansion velocity is $11 \mathrm{~km} \mathrm{~s}^{-1}$. The high-velocity bipolar outflow is oriented perpendicular to this disk, with a velocity of at least $45 \mathrm{~km} \mathrm{~s}^{-1}$. The emission at intermediate velocities in the range $15-25 \mathrm{~km} \mathrm{~s}^{-1}$ from the systemic velocity may correspond to the material punched out of the disk by the bipolar outflow. The similarity between $\pi^{1}$ Gru and V Hya suggests that a common mechanism might be at work in creating the disk-outflow structure of AGB stars. Our result provides further evidence that the bipolar structures seen in PNs and PPNs may be produced during the AGB stage.

We thank all the SMA staff at Cambridge, Hawaii, and Taipei for their help during the observation. We are grateful to G. R. Knapp for providing us the CSO single-dish data. This work is supported in part by Academia Sinica and by grants from the National Science Council of Taiwan (NSC) to D.-V-T. (NSC 94-2112-M-001-008), J. L., and S. K. P.-J. C. acknowledges a graduate fellowship from NSC. This research has made use of NASA's Astrophysics Data System, as well as the SIMBAD database operated at CDS, Strasbourg, France.
Abia, C., \& Wallerstein, G. 1998, MNRAS, 293, 89

Ake, T. B., \& Johnson, H. R 1992, AAS Meeting, 180, 37.08

Balick, B., \& Frank, A. 2002, ARA\&A, 40, 439

Bujarrabal, V., Alcolea, J., Neri, R., \& Grewing, M. 1997, A\&A, 320, 540

Feast, M. W. 1953, MNRAS, 113, 510

Goldreich, P., \& Scoville, N. 1976, ApJ, 205, 144

Hirano, N., et al. 2004, ApJ, 616, L43

Ho, P. T. P., Moran, J. M., \& Lo, K. Y. 2004, ApJ, 616, L1

Iben, I., Jr., \& Livio, M. 1993, PASP, 105, 1373

Kahane, C., Audinos, P., Barnbaum, C., \& Morris, M. 1996, A\&A, 314, 871

Knapp, G. R., Jorissen, A., \& Young, K. 1997, A\&A, 326, 318

Knapp, G. R., Young, K., \& Crosas, M. 1999, A\&A, 346, 175 (K99)

Kwan, J., \& Linke, R. A. 1982, ApJ, 254, 587

Kwok, S. 1993, ARA\&A, 31, 63

Kwok, S., Hrivnak, B. J., \& Su, K. Y. L. 2000, ApJ, 544, L149

Kwok, S., Hrivnak, B. J., Zhang, C. Y., \& Langill, P. L. 1996, ApJ, 472, 287

Kwok, S., Su, K. Y. L., \& Hrivnak, B. J. 1998, ApJ, 501, L117

\section{REFERENCES}

Mastrodemos, N., \& Morris, M. 1998, ApJ, 497, 303

1999, ApJ, 523, 357

Matt, S., Balick, B., Winglee, R., \& Goodson, A. 2000, ApJ, 545, 965

Morris, M. 1987, PASP, 99, 1115

Neri, R., Kahane, C., Lucas, R., Bujarrabal, V., \& Loup, C. 1998, A\&AS, 130, 1

Olofsson, H., \& Nyman, L.-Å. 1999, A\&A, 347, 194

Perryman, M. A. C., et al. 1997, A\&A, 323, L49

Piccirillo, J. 1980, MNRAS, 190, 441

Proust, D., Ochsenbein, F., \& Petterson, B. R. 1981, A\&AS, 44, 179

Sahai, R. 1992, A\&A, 253, L33 (S92)

Scalo, J. M., \& Ross, J. E. 1976, A\&A, 48, 219

Theuns, T., Boffin, H. M. J., \& Jorissen, A. 1996, MNRAS, 280, 1264

Van Eck, S., Jorissen, A., Udry, S., Mayor, M., \& Pernier, B. 1998, A\&A, 329, 971

Young, K., Phillips, T. G., \& Knapp, G. R. 1993, ApJ, 409, 725 